\title{
Management of malperfusion syndrome in acute type A aortic intramural hematoma
}

\author{
Elizabeth L. Norton ${ }^{1}$, David M. Williams ${ }^{2}$, Karen M. Kim ${ }^{3}$, Xiaoting Wu ${ }^{3}$, Minhaj S. Khaja ${ }^{2}$, \\ Himanshu J. Patel ${ }^{3}$, G. Michael Deeb ${ }^{3}$, Bo Yang ${ }^{3}$ \\ ${ }^{1}$ Creighton University School of Medicine, Omaha, NE, USA; ${ }^{2}$ Department of Radiology, ${ }^{3}$ Department of Cardiac Surgery, Michigan Medicine, Ann \\ Arbor, MI, USA \\ Correspondence to: Bo Yang, MD, PhD. Department of Cardiac Surgery, Michigan Medicine, 1500 East Medical Center Drive, 5155 Frankel \\ Cardiovascular Center, Ann Arbor, MI 48109, USA. Email: boya@med.umich.edu.
}

Background: We report the outcomes of acute type A aortic intramural hematoma (ATAAIMH) with malperfusion treated with endovascular intervention and delayed open aortic repair.

Methods: Between April 1998 and April 2018, 644 patients were treated at our institution with an acute type A aortic dissection (ATAAD) or ATAAIMH, 82 (13\%) had intramural hematomas (IMHs) including $12(15 \%)$ with malperfusion syndrome (MPS) and 70 (85\%) without MPS (no MPS). Data was obtained through medical record review, the Society of Thoracic Surgeons data elements, and the National Death Index database.

Results: Both MPS and No MPS groups had similar comorbidities including coronary artery disease, hypertension, diabetes, and peripheral vascular disease; however, those with MPS were sicker on admission with higher rates of acute renal failure $(50 \%$ vs. $1 \%, \mathrm{P}<0.0001)$ and acute paralysis $(17 \%$ vs. $0 \%, \mathrm{P}=0.02)$. Patients with MPS amenable to endovascular reperfusion $(\mathrm{n}=10)$ underwent endovascular fenestration/ stenting and delayed aortic repair. Those with cerebral or coronary MPS ( $n=2)$ and those without MPS (n=70) underwent emergent open aortic repair. Of the ten patients undergoing fenestration/stenting, seven went on to aortic repair, one survived to discharge without aortic repair, one died from aortic rupture on hospital day 34 , and one died from organ failure prior to aortic repair. Following endovascular fenestration/stenting or aortic repair, all patients with MPS had higher in-hospital mortality (17\% vs. 0\%), P=0.02. Following aortic repair, patients with MPS had more postoperative sepsis and longer postoperative length of stay (all $\mathrm{P}<0.05)$. However, both groups had a $0 \%$ operative mortality (including in-hospital and 30-day mortality following aortic repair). The 5-year survival of all ATAAIMH patients was $79 \%$. The 2 -year survival was significantly better in the No MPS group (94\% vs. 62\%, P=0.006).

Conclusions: ATAAIMH with MPS can be effectively managed with upfront endovascular fenestration/ stenting followed by delayed aortic repair.

Keywords: Aortic dissection; malperfusion syndrome (MPS); aortic intramural hematoma (IMHs)

Submitted Mar 26, 2019. Accepted for publication Jul 11, 2019.

doi: 10.21037 /acs.2019.07.03

View this article at: http://dx.doi.org/10.21037/acs.2019.07.03

\section{Introduction}

The reported incidence of acute type A aortic intramural hematoma (ATAAIMH) varies between $3.5-38 \%$ of type A aortic syndromes (1-8), with differences in incidence and treatment strategies between Eastern and Western countries. The 2010 AHA guidelines state that intramural hematomas
(IMHs) involving the ascending aorta have a high, early risk of complication and death; therefore, should be managed surgically (9); however, others, particularly in eastern countries, have shown favorable outcomes with medical management $(4,10,11)$. ATAAIMH has similar risk factors and complications as classic acute type A aortic dissection 

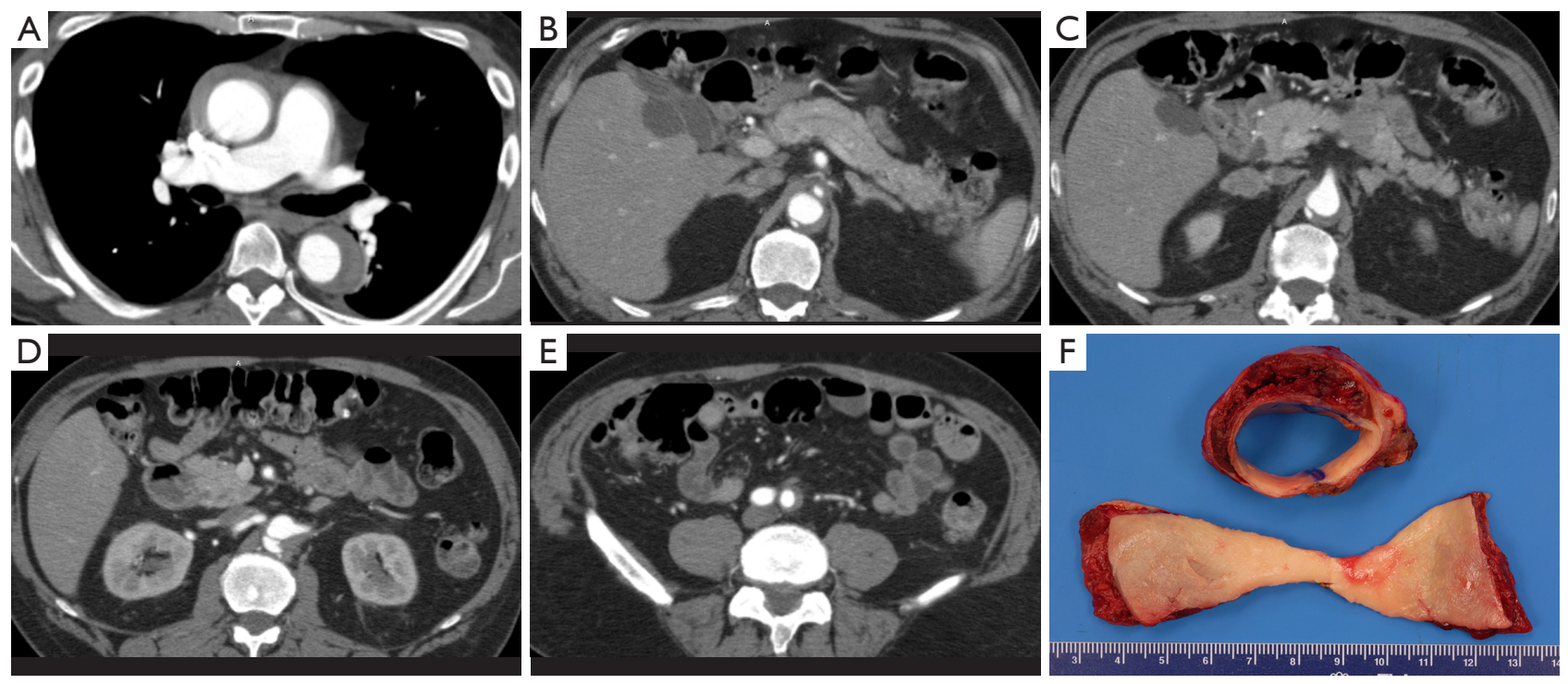

Figure 1 Computed tomography angiography of intramural hematoma (IMH) in the ascending and descending aorta (A), and static obstruction due to IMH of aortic branch vessels, including the celiac artery (B), superior mesenteric artery (C), left renal artery (D), left common iliac artery (E), and intraoperative finding of ascending $\mathrm{IMH}$ and intimal tear (F).

(ATAAD), including presence of malperfusion syndrome (MPS), which is associated with increased mortality. Management of MPS in classic ATAAD remains debated, but upfront endovascular reperfusion via transcatheter techniques and delayed aortic repair has shown favorable outcomes (12-15). Although ATAAIMH is less often complicated by MPS $(1-3,7,8,16)$, there is a paucity of information regarding MPS in the setting of ATAAIMH.

In this study, we report the outcomes of ATAAIMH patients with visceral or extremity MPS (malperfusion with tissue/organ necrosis and end-organ dysfunction) treated with upfront endovascular reperfusion through fenestration/ stenting of critically malperfused organ system(s) by interventional radiology (IR) followed by aortic repair at resolution of organ failure (12-15) and without visceral or extremity MPS treated with immediate aortic repair.

\section{Methods}

This study was approved by the Institutional Review Board at the University of Michigan, Michigan Medicine (Ann Arbor, MI) and was in compliance with Health Insurance Portability and Accountability Act regulations.

\section{Study population and data collection}

From April 1998 to April 2018, 644 patients underwent upfront endovascular fenestration/stenting for MPS or emergent aortic repair of an ATAAD or IMH (ATAAD/ IMH defined as onset within 14 days of admission); 82 (13\%) with a type A IMH (defined as IMH without a double lumen in the ascending aorta) (Figure 1), comprised the focus of this study. Among those with ATAAIMH, 12 presented with MPS and 70 presented without MPS. This cohort includes patients with small and sometimes subtle intimal tears with no or minimal sub-intimal contrast spillage and with or without segments of descending thoracic or abdominal aorta with patent false lumens.

Investigators leveraged Society of Thoracic Surgeons data elements from the University of Michigan Cardiac Surgery Data Warehouse to identify the cohort and determine preoperative, operative, and post-operative characteristics. Electronic medical record review was conducted to confirm ATAAIMH diagnosis and to supplement data collection. Investigators utilized the National Death Index database through December 31, 2015 (17), medical record review, and phone call survey (January 2018) to obtain long-term survival.

\section{Diagnosis and management of MPS in ATAAIMH}

MPS is different from malperfusion. A diagnosis of MPS entails end-organ dysfunction and requires both clinical and laboratory features (neurologic deficit, abdominal 
pain, bloody diarrhea, tenderness to palpation, elevated lactate, troponins, liver enzymes, metabolic acidosis, etc.) in addition to radiographic demonstration of decreased or absent blood flow to a vascular territory (brain, coronary, liver, bowel, kidneys, extremities, etc.) (Figure 1). Patients with endovascular-amenable MPS (visceral and extremities, $\mathrm{n}=10$ ) and without evidence of aortic rupture or cardiac tamponade (indications for immediate aortic repair) were treated with upfront endovascular fenestration/stenting prior to aortic repair. Patients with cerebral $(n=1)$ and coronary $(\mathrm{n}=1)$ MPS were managed with emergent open repair as previously described $(14,15)$.

All fenestration/stenting was performed percutaneously by IR in the angiography suite or hybrid room at the University of Michigan. In aortic IMH, the false lumen is thrombosed, and dynamic collapse of adjacent true lumen does not occur. Instead, localized false lumen thrombosis can result in coarctation-like narrowing of adjacent true lumen with static obstruction. Treatment strategy in this clinical situation consists of fenestration at the rostral margin of the false lumen thrombosis (to establish transluminal flow and prevent further retrograde thrombosis of the false lumen) and stenting of the true lumen. Two precautions should be observed. The stent should be post-dilated with extreme caution or not at all, because balloon-occlusion of the true lumen adjacent to thrombosed false lumen creates total aortic occlusion, with risk of proximal aortic rupture or extension of the false lumen. Second, stenting should begin at the narrowest segment of the coarctation like narrowing and proceed proximally and distally with sufficient stentoverlap to avoid watermelon-seed migration of the stents. Patients with type A IMH may have segments of patent false lumen in the descending thoracic or abdominal aorta, in which the adjacent true lumen is subject to collapse and dynamic obstruction and malperfusion. Endovascular fenestration and stenting are performed here as described elsewhere $(14,15)$. Initially, treatable MPS is confirmed angiographically by a significant pressure gradient $(>15 \mathrm{mmHg}$ ) between the ascending aorta true lumen and a branch artery $(12-14,18)$. Blood pressure of the aorta and its branches were measured before and after aortic fenestration/ stenting. Dissected branch arteries with a significant gradient are inspected by $6 \mathrm{Fr}$ intravascular ultrasound (IVUS) to determine the end-point of dissection and to distinguish between false lumen thrombus requiring a stent and distal true lumen thrombus requiring thrombectomy or lysis. If a pressure gradient remained between the ascending aorta and a branch vessel (such as the superior mesenteric artery) after correction of obstruction by aortic fenestration/ stenting, then branch vessel stenting, thrombolysis, or suction thromboembolectomy was performed. Among the 10 patients treated for endovascular-amenable MPS, $2(17 \%)$ had aortic fenestration, 4 (33\%) had aortic stenting, and $6(50 \%)$ had branch vessel stenting. In dissected vessels with thrombosed false lumens, gradients after stenting might exceed $15 \mathrm{mmHg}$, but as long as absolute perfusion pressure was viable, i.e., $>60 \mathrm{mmHg}$, post-dilation of stents was not performed.

Following endovascular reperfusion, patients were evaluated by general and/or vascular surgery for bowel and/or extremity ischemia respectively to determine if an exploratory laparotomy or fasciotomies were needed. Of the 10 patients, none underwent a fasciotomy or amputation for extremity malperfusion and 1 patient underwent exploratory laparotomy for possible bowel ischemia, which was negative. After patients recovered from organ failure or shock and surgeons assessed that they could tolerate aortic operation, they underwent delayed aortic repair. The median waiting time between endovascular fenestration/stenting and aortic repair was 2 [interquartile range (IQR): 1, 8] days.

\section{Surgical techniques}

Two patients underwent thoracic endovascular aortic repair (TEVAR) for a retrograde type A aortic dissection. All other patients underwent open aortic repair via a standard median sternotomy. Indications for root replacement included an intimal tear at the aortic root, root diameter $\geq 4.5 \mathrm{~cm}$, connective tissue disease, and unrepairable aortic valve pathology (19). Root replacement was performed as a total root (BioBentall with Freestyle $)(n=7)$, inclusion root with a Freestyle bioprosthesis $(n=4)$, or David valve-sparing aortic root replacement $(n=2)$; all of which are current practice in our group. If criteria were not met for aortic root replacement, aortic root repair was performed $(n=52)$ by preserving the aortic valve and all sinuses of Valsalva based on surgeons' preference as previously described (20-22). With aortic valve pathology in the absence of aortic root pathology, an isolated aortic valve replacement was performed $(\mathrm{n}=1)$. Indications for zone $1-3$ arch replacement included an arch aneurysm $>4 \mathrm{~cm}$, intimal tear located in the arch which could not be resected by a hemiarch replacement, or dissection of arch branch vessels with malperfusion (23). Extent of arch procedure, hemiarch, zone 1,2 , or 3 arch replacement was based on arch pathology as described above. Zone 1 arch replacement divides the arch 
between the innominate and left common carotid (LCC) arteries with reimplantation of the innominate artery; zone 2 arch replacement divides the arch between LCC and left subclavian arteries with reimplantation of the innominate and LCC arteries; and zone 3 arch replacement (total arch replacement) divides the arch distal to the left subclavian artery with reimplantation of all three head vessels. All arch branch vessels were reimplanted individually to branch grafts. In the past 5 years, a frozen elephant trunk (cTAG $10 \mathrm{~cm}$; Gore Associates, Flagstaff, AZ, USA) has been used in patients with a tear in the proximal descending thoracic aorta or, if the true lumen of the descending thoracic aorta was severely compressed. The stented component of the frozen elephant trunk was placed into the true lumen of the descending thoracic aorta distal to the left subclavian artery as previously described (23). Both retrograde and antegrade cerebral perfusion (ACP), with a shift toward antegrade in recent years, and hypothermic circulatory arrest (HCA) were used for arch repair. Patients were cooled to $18{ }^{\circ} \mathrm{C}$ before 2012 and to $24-28^{\circ} \mathrm{C}$ since 2012 for ACP.

\section{Statistical analysis}

The initial analysis provided descriptive information on the demographic, clinical, and surgical characteristics. Continuous variables were summarized by median (25th percentile, 75 th percentile) and categorical variables were reported as $\mathrm{n}(\%)$ in frequency tables. Univariate comparisons between MPS and no MPS groups were performed using Chi-square tests or fisher exact tests for categorical data and Wilcoxon rank sum tests for continuous data. Crude survival curves since admission were estimated using the non-parametric Kaplan-Meier method. Log-Rank test was used to compare the survival between groups. All statistical calculations used SAS 9.4 (SAS Institute, Cary, NC, USA) and were considered significant at $\mathrm{P}<0.05$.

\section{Results}

\section{Demographics and preoperative data}

The cohort $(\mathrm{n}=82)$ had a median age of 63.5 years with slight male predominance (57\%). The incidence of MPS in ATAAIMH was $15 \%$. Patients with $(n=12)$ and without $(\mathrm{n}=70)$ MPS had similar comorbidities including coronary artery disease (CAD), hypertension, chronic obstructive pulmonary disease (COPD), smoking status, diabetes, connective tissue disorder, and aortic insufficiency; all
$\mathrm{P}>0.05$. However, patients with MPS were sicker on presentation with more acute paralysis $(17 \%$ vs. $0 \%$, $\mathrm{P}=0.02)$ and acute renal failure $(50 \%$ vs. $1 \%, \mathrm{P}<0.0001)$ than those without MPS (Table 1). MPS consisted of coronary $(8 \%)$, cerebral $(8 \%)$, spinal cord $(17 \%)$, celiac/hepatic $(17 \%)$, mesenteric (33\%), renal (50\%), and lower extremity (25\%), with multiple vascular beds often affected (Table 2).

\section{IR procedure and/or intraoperative data}

Of the 12 patients who presented with MPS, 10 underwent upfront endovascular fenestration/stenting (Tables 2,3) and 2 underwent immediate aortic repair due to MPS not amenable to endovascular reperfusion (coronary or cerebral) but able to be resolved with open aortic repair. Following endovascular fenestration/stenting, two patients died before aortic repair- either from organ failure $(n=1)$ or aortic rupture $(n=1)$. One patient survived to discharge without aortic repair.

Eventually, there were 9 patients with MPS and 70 patients without MPS who had open aortic repair. Patients with MPS had more acute renal failure before open repair, $\mathrm{P}<0.004$. (Table S1). Both groups underwent similar aortic root and arch procedures, with similar cardiopulmonary bypass $(\mathrm{CPB})$, cross-clamp, and HCA times, and intraoperative transfusion requirements (Table $S 2)$.

\section{Post-IR procedure and/or operative outcomes}

The MPS group had similar rates of post-procedural complications (any complications after endovascular fenestration/stenting or aortic repair) as the No MPS group. The MPS group had longer total length of stay (LOS) (23.5 vs. 11 days, $\mathrm{P}=0.006)$ and a higher in-hospital mortality ( $17 \%$ vs. $0 \%, \mathrm{P}=0.02)$ (Table 4). Among those undergoing aortic repair (total, $n=79$ : MPS, n=9; no MPS, $\mathrm{n}=70$ ), those with MPS had more sepsis postoperatively and had longer postoperative LOS (16 vs. 10 days, $\mathrm{P}=0.02$ ). Both groups had an operative mortality following aortic repair of $0 \%$, which includes in-hospital mortality and/or 30-day mortality after surgery (Table 5).

\section{Mid-term survival}

The total follow-up was 312 patient-years and the mean follow-up time was 3.8 years. Ten (12\%) patients were lost to follow-up and were censored to the date of last known status. The overall 5- and 10-year survival for all ATAAIMH 


\begin{tabular}{|c|c|c|c|c|}
\hline Age on admission [years] & $63.5[56,73]$ & $62[55,73.5]$ & $64[56,73]$ & 0.83 \\
\hline BMI & $28.1(24.3,31.6)$ & $30.4(27.5,37.1)$ & $27.6(23.7,30.7)$ & 0.02 \\
\hline Gender, male & $47[57]$ & $8[67]$ & $39[56]$ & 0.48 \\
\hline History of MI & $5[6]$ & $0[0]$ & $5[7]$ & 1.0 \\
\hline Hypertension & 67 [82] & 11 [92] & $56[80]$ & 0.45 \\
\hline COPD & 10 [12] & $2[17]$ & $8[11]$ & 0.64 \\
\hline Smoking status & & & & 0.73 \\
\hline Current smoker & 19 [23] & $3[25]$ & 16 [23] & \\
\hline Diabetes & $9[11]$ & $2[17]$ & $7[10]$ & 0.31 \\
\hline Creatinine on admission (mg/dL) & $0.9(0.8,1.1)$ & $1.1(0.9,1.4)$ & $0.9(0.8,1.1)$ & 0.03 \\
\hline Chronic kidney disease & $0[0]$ & $0[0]$ & $0[0]$ & - \\
\hline History of CVA & $4[5]$ & $0[0]$ & $4[6]$ & 1.0 \\
\hline PVOD & 10 [12] & $2[17]$ & $8[11]$ & 0.64 \\
\hline Connective tissue disorder & $3[4]$ & $0[0]$ & $3[4]$ & 1.0 \\
\hline Ejection Fraction & $56[55,60]$ & $55[52.5,60]$ & $60[55,60]$ & 0.22 \\
\hline Severe & 2 [2] & $0[0]$ & $2[3]$ & \\
\hline Cardiogenic shock & $8[10]$ & $1[8]$ & $7[10]$ & 1.0 \\
\hline Acute stroke & $2[2]$ & $1[8]$ & $1[1]^{*}$ & 0.27 \\
\hline Acute paralysis & $2[2]$ & $2[17]$ & $0[0]$ & 0.02 \\
\hline Acute MI & $1[1]$ & $1[8]$ & $0[0]$ & 0.15 \\
\hline Acute renal failure & 7 [9] & $6[50]$ & $1[1]$ & $<0.01$ \\
\hline
\end{tabular}

Data presented as median (interquartile range) for continuous variables and number (percentage) for categorical variables. *, no MPS acute stroke: patient suffered anoxic brain injury due to cardiac arrest with ROSC preoperatively. MPS, malperfusion syndrome; BMI, body mass index; CAD, coronary artery disease; COPD, chronic obstructive pulmonary disease; CVA, cerebrovascular accident; PVOD, peripheral vascular occlusive disease; MI, myocardial infarction. 


\begin{tabular}{|c|c|c|c|c|}
\hline Malperfusion management & All patients $(n=82)$ & MPS (n=12) & No MPS $(n=70)$ & $P$ value \\
\hline Malperfusion syndrome & $12[15]$ & 12 [100] & $0[0]$ & $<0.01$ \\
\hline Coronary & $1[1]$ & $1[8]$ & $0[0]$ & 0.15 \\
\hline Cerebral & $1[1]$ & $1[8]$ & $0[0]$ & 0.15 \\
\hline Spinal cord & 2 [2] & $2[17]$ & $0[0]$ & 0.02 \\
\hline Celiac/hepatic & 2 [2] & $2[17]$ & $0[0]$ & 0.02 \\
\hline Mesenteric & $4[5]$ & 4 [33] & $0[0]$ & $<0.01$ \\
\hline Renal & $6[7]$ & $6[50]$ & $0[0]$ & $<0.01$ \\
\hline Lower extremity & $3[4]$ & $3[25]$ & $0[0]$ & $<0.01$ \\
\hline \multicolumn{5}{|l|}{ Management } \\
\hline Emergent aortic repair & 72 [88] & $2[17]$ & 70 [100] & $<0.01$ \\
\hline IR & $10[12]$ & $10[83]$ & $0[0]$ & $<0.01$ \\
\hline Aortic fenestration & 2 [2] & $2[17]$ & - & - \\
\hline Aortic stenting & $4[5]$ & 4 [33] & - & - \\
\hline Branch vessel fenestration & $0[0]$ & $0[0]$ & - & - \\
\hline Branch vessel stenting & $6[7]$ & $6[50]$ & - & - \\
\hline Time from admission to IR [days] & - & $0[0,1]$ & - & - \\
\hline Aortic repair & 79 [96] & $9[75]$ & $70[100]$ & $<0.01$ \\
\hline Time from admission to aortic repair [days] & $0[0,1]$ & $3[1,7]$ & $0[0,1]$ & $<0.01$ \\
\hline Time from IR to aortic repair [days] & - & $2[1,8]$ & - & - \\
\hline No aortic repair & $3[4]$ & $3[25]$ & $0[0]$ & $<0.01$ \\
\hline Death due to organ failure & $1[1]$ & $1[8]$ & - & - \\
\hline Death due to aortic rupture & $1[1]$ & $1[8]$ & - & - \\
\hline Survival to discharge & $1[1]$ & $1[8]$ & - & - \\
\hline
\end{tabular}

Data presented as median (interquartile range) for continuous variables and number (percentage) for categorical variables. MPS, malperfusion syndrome; IR, interventional radiology.

\begin{tabular}{|c|c|c|c|c|c|}
\hline $\begin{array}{l}\text { Level of aortic } \\
\text { fenestration/stenting }\end{array}$ & Aortic fenestration & Aortic stenting & $\begin{array}{l}\text { Branch vessel } \\
\text { thrombolysis }\end{array}$ & $\begin{array}{l}\text { Branch vessel thrombectomy/ } \\
\text { embolectomy }\end{array}$ & $\begin{array}{l}\text { Branch vessel } \\
\text { stenting }\end{array}$ \\
\hline Arch branch vessel & - & - & 0 & 0 & 0 \\
\hline Descending thoracic & 0 & 1 & - & - & - \\
\hline Supraceliac & 1 & 1 & - & - & - \\
\hline Celiac & 0 & 0 & 0 & 0 & 1 \\
\hline Supramesenteric & 1 & 1 & - & - & - \\
\hline Mesenteric & 0 & 0 & 0 & 0 & 3 \\
\hline Suprarenal & 0 & 0 & - & - & - \\
\hline Transrenal & 0 & 0 & - & - & - \\
\hline Renal & - & - & 0 & 0 & 1 \\
\hline Infrarenal & 0 & 3 & - & - & - \\
\hline Iliac & - & - & 1 & 0 & 3 \\
\hline
\end{tabular}




\begin{tabular}{|c|c|c|c|c|}
\hline Outcomes & All patients $(n=82)$ & MPS $(n=12)$ & No MPS $(n=70)$ & $P$ value \\
\hline Reoperation for bleeding & $5[6]$ & $0[0]$ & $5[7]$ & 1.0 \\
\hline Tamponade & $0[0]$ & $0[0]$ & $0[0]$ & - \\
\hline Post-intervention MI & $1[1]$ & $1[8]$ & $0[0]$ & 0.15 \\
\hline Atrial fibrillation & $36[44]$ & $6[50]$ & $30[43]$ & 0.65 \\
\hline New-onset CVA & $6[7]$ & $0[0]$ & $6[9]$ & 0.59 \\
\hline New-onset paralysis & $1[1]$ & $0[0]$ & $1[1]$ & 1.0 \\
\hline Pneumonia & $14[17]$ & 4 [33] & $10[14]$ & 0.21 \\
\hline Reintubation & $4[5]$ & $0[0]$ & $4[4]$ & 1.0 \\
\hline Tracheostomy & $3[4]$ & $1[8]$ & $2[3]$ & 0.38 \\
\hline New-onset acute renal failure & $8[10]$ & $0[0]$ & $8[11]$ & 0.60 \\
\hline Requiring dialysis & $3[4]$ & $0[0]$ & $3[4]$ & 1.0 \\
\hline Total LOS [days] & $12[8,23]$ & $23.5[16.5,34.5]$ & $11[8,18]$ & $<0.01$ \\
\hline In-hospital mortality & $2[2]$ & $2[17]$ & $0[0]$ & 0.02 \\
\hline
\end{tabular}

Data presented as median (interquartile range) for continuous variables and number (percentage) for categorical variables. In the MPS group, any complications after IR procedures or AR were recorded as outcomes. In the No MPS group, any complications after open repair were recorded as outcomes. IR, interventional radiology; AR, aortic repair; MPS, malperfusion syndrome; MI, myocardial infarction; CVA, cerebrovascular accident; LOS, length of stay.

\begin{tabular}{|c|c|c|c|c|}
\hline Outcomes after open aortic repair & All patients $(n=79)$ & MPS $(n=9)$ & No MPS $(n=70)$ & $P$ value \\
\hline Reoperation for bleeding & $5[6]$ & $0[0]$ & $5[7]$ & 1.0 \\
\hline Tamponade & $0[0]$ & $0[0]$ & $0[0]$ & - \\
\hline Postoperative MI & $0[0]$ & $0[0]$ & $0[0]$ & - \\
\hline Atrial fibrillation & $35[44]$ & $5[56]$ & 30 [43] & 0.50 \\
\hline DSWI & $0[0]$ & $0[0]$ & $0[0]$ & - \\
\hline Sepsis & $3[4]$ & $2[22]$ & $1[1]$ & 0.03 \\
\hline New-onset CVA & $6[8]$ & $0[0]$ & 6 [9] & 1.0 \\
\hline New-onset paralysis & $1[1]$ & $0[0]$ & $1[1]$ & 1.0 \\
\hline Pneumonia & $13[16]$ & $3[33]$ & $10[14]$ & 0.16 \\
\hline Reintubation & $4[5]$ & $0[0]$ & $4[6]$ & 1.0 \\
\hline Prolonged ventilation & 52 [66] & 8 [89] & 44 [63] & 0.15 \\
\hline Tracheostomy & $3[4]$ & $1[11]$ & $2[3]$ & 0.31 \\
\hline New-onset renal failure & $8[10]$ & $0[0]$ & $8[11]$ & 0.59 \\
\hline Requiring dialysis & $3[4]$ & $0[0]$ & $3[4]$ & 1.0 \\
\hline Gl complications & $8[10]$ & $1[11]$ & $7[10]$ & 1.0 \\
\hline Postoperative LOS [days] & $11[7,18]$ & $16[14,20]$ & $10[7,18]$ & 0.02 \\
\hline Intraoperative mortality* & $0[0]$ & $0[0]$ & $0[0]$ & - \\
\hline In-hospital mortality & $0[0]$ & $0[0]$ & $0[0]$ & - \\
\hline 30-day mortality & $0[0]$ & $0[0]$ & $0[0]$ & - \\
\hline
\end{tabular}




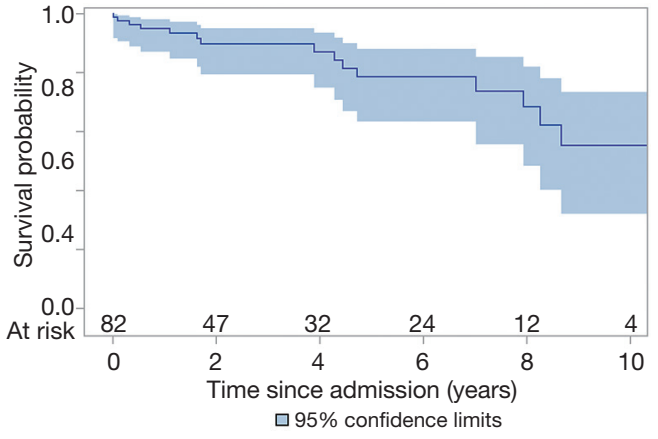

Figure 2 Overall mid-term survival of all acute type A aortic intramural hematoma patients $(n=82)$ since hospital admission.

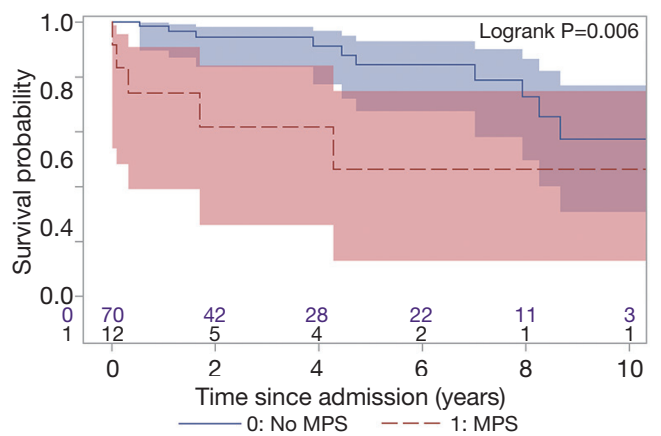

Figure 3 Overall mid-term survival of all acute type A aortic intramural hematoma patients with MPS $(n=12) v s$. no MPS $(n=70)$. MPS, malperfusion syndrome.

patients was $79 \%$ and $55 \%$, respectively (Figure 2). Twoyear survival was significantly better in the No MPS group (94\% vs. 62\%, $\mathrm{P}=0.006$ ) (Figure 3).

\section{Discussion}

In this study, we managed ATAAIMH with visceral/ extremity MPS with endovascular fenestration/stenting followed by aortic repair; and immediate open aortic repair for patients with isolated cerebral or coronary MPS. The overall in-hospital mortality of all ATAAIMH patients was $2.4 \%$, with an in-hospital mortality of $17 \%$ in ATAAIMH patients with MPS, which is significantly higher than that in ATAAIMH patients without MPS ( $0 \%$ in-hospital mortality); and the 2-year survival was significantly worse (62\% vs. 94\%) in patients with MPS. All patients with inhospital mortality had MPS.

The development of malperfusion and MPS in ATAAIMH remains an incompletely answered question.
Additionally, the mechanism of ATAAIMH is unknown: it has been suggested that it could originate from rupture of the vasa vasorum $(24,25)$ or thrombosed false lumen of aortic dissection. More and more evidence point toward the latter with an intimal tear identified in IMH cases $(3,26,27)$. When we operate on ATAAIMH, the thrombosed false lumen of the ascending aorta is identified in all cases. Whether the ATAAIMH developed from ruptured vasa vasorum or intimal tear, when patients develop fullblown ATAAIMH, the thrombosed false lumen can extend into any branch of the aorta, causing malperfusion and subsequent MPS. However, the incidence of MPS in ATAAIMH (15\%) is lower than that in ATAAD patients (30\%) (14). In ATAAIMH patients, when the false lumen is completely thrombosed throughout the aorta, there can be no dynamic obstruction of the aortic branches or dynamic malperfusion of end-organs. Most of the malperfusion in ATAAIMH is static and occurs when the aortic branches are involved with IMH (Figure 1), and cannot reliably be resolved by open aortic repair. This is different from malperfusion in ATAAD, which can be dynamic or static. Therefore, malperfusion with MPS (tissue/organ necrosis and end-organ dysfunction) in ATAAIMH may require revascularization to resolve malperfusion before aortic repair, since proximal aortic repair does not significantly alter flow or increase perfusion pressure in the descending aorta and therefore, cannot predictably resolve static malperfusion. As noted above, type A IMH as well as classic type A dissection can be accompanied by segmental false lumen thrombosis distally, such as at the aortic bifurcation, and in these cases, fenestration is used to arrest retrograde false lumen thrombosis, with stenting necessary to resolve any persisting static obstruction. In summary, endovascular fenestration/stenting can treat the anatomical spectrum of MPS accompanying IMH, and is the treatment of choice except when there is significant coronary or neurovascular compromise.

Due to the potential risk of aortic rupture, is it safe to perform upfront endovascular fenestration/stenting followed by delayed aortic repair? Multiple studies recommend initial medical management of ATAAIMH $(1,4,6,11)$ and delayed ("timely") aortic repair (within first 3 days $(2,28)$ in stable patients with ATAAIMH-these cite favorable operative outcomes $(2,6,28)$. However, others report a high incidence of early rupture in ATAAIMH vs. classic ATAAD (29-31), suggesting delaying aortic repair may be detrimental. Over the past 20 years, we have been managing ATAAIMH with emergent aortic 
repair, unless complicated by endovascular-amenable MPS, which demands immediate revascularization given no signs of aortic rupture or cardiac tamponade. The median [IQR] time from admission to aortic repair was longer in the MPS group, 3 days $[1,7]$ vs. $0[0,1]$ due to the upfront endovascular reperfusion and recovery prior to aortic repair. The median time between endovascular reperfusion and aortic repair was 2 days (Table 2). In total, out of 10 patients with MPS and delayed aortic repair, we had only one patient rupture prior to aortic repair. The rupture occurred 34 days following admission/ endovascular reperfusion, and this patient was very sick with mesenteric and renal MPS which required stenting of the superior mesenteric artery to correct static mesenteric malperfusion, which was not amenable to open aortic repair. This patient required dialysis after endovascular reperfusion and three exploratory laparotomies which revealed no gross necrosis of intestines but intraabdominal abscess. This case also occurred early in our experience with this management strategy. In more recent years [2008-2017], experience and adjustments (aggressive blood pressure control, better care in the intensive care unit, and earlier aortic repair) in this management strategy $(14,15)$ has led to a decrease in rupture rate $(16 \%$ to $4 \%)$ in all ATAAD patients with MPS waiting for delayed open aortic repair. We think it is reasonable to perform endovascular fenestration/stenting first followed by delayed aortic repair for ATAAIMH patients with IR amenable MPS if there is no cardiac tamponade, since the risk of aortic rupture is low.

The mid-term survival of all ATAAIMH patients was favorable, $79 \%$ at 5 years and $55 \%$ at 10 years, which was similar to ATAAD patients (14). However, the late survival was very poor in the ATAAIMH with MPS patients with $62 \%$ survival at 2 years, mainly due to in-hospital mortality. Because the sample size is small in the MPS group, it is hard to make a meaningful conclusion regarding midterm survival. On the other hand, emergent aortic repair worked very well in the ATAAIMH patients without MPS, with $0 \%$ in-hospital or operative mortality, and $84 \% 5$-year survival which was similar to other reports $(1,32)$. We would recommend emergent open aortic repair for ATAAIMH with no IR-amenable MPS.

In summary, ATAAIMH carries significant morbidity and mortality, similar to classic ATAAD $(1,8)$, especially when complicated by MPS $(33,34)$. This was shown in our study's MPS group, where $17 \%$ in-hospital mortality occurred. Although MPS is less frequently seen in ATAAIMH compared to classic ATAAD $(1-3,7,8,16)$, it still occurs and yields a worse prognosis, so it is important for clinicians to recognize. In ATAAIMH without MPS, emergent open aortic repair should be performed since the operative mortality is very low in both our study ( $0 \%$ in-hospital mortality) and other studies $(3,32,35)$ compared to $40 \%$ mortality in ATAAIMH patients managed only medically (8). In ATAAIMH patients with IR-amenable MPS, i.e., visceral or extremity MPS, we recommend endovascular reperfusion through fenestration/stenting to resolve visceral and extremity malperfusion followed by open aortic repair when the patient can tolerate the aortic procedure.

Our study is limited by its single-center and retrospective nature. The follow-up time is still relatively short and the sample size of patients with MPS is relatively small. The vast majority of cases were performed by aortic surgeons and we are one of the few centers using IR for upfront endovascular reperfusion through fenestration/stenting to manage visceral and extremity MPS prior to open aortic repair; therefore, our experience may not apply to all centers managing ATAAIMH patients. Additional studies with larger sample sizes regarding MPS in ATAAIMH would be helpful.

In conclusion, ATAAIMH patients with visceral/ extremity MPS could be managed with initial endovascular reperfusion of the vascular territories affected by malperfusion and delayed open aortic repair as our experience shows it achieves favorable short- and mid-term outcomes in this complex disease.

\section{Acknowledgments}

The authors would like to thank the support from the Data Warehouse-Department of Cardiac Surgery led by Dr. Donald Likosky, including Jeremy Wolverton, Amy Geltz, Mary Barry, Brett Cross, Mary Ryzak, and other team members as well as the support from the Department of Cardiac Surgery and the Frankel Cardiovascular Center at the University of Michigan.

Funding: Dr. Yang is supported by the NHLBI of NIH K08HL130614 and R01HL141891, Phil Jenkins and Darlene \& Stephen J. Szatmari Funds. Dr. Patel is supported by the Joe D. Morris Collegiate Professorship, the David Hamilton Fund, and the Phil Jenkins Breakthrough Fund in Cardiac Surgery. Dr. Deeb is supported by the Herbert Sloan Collegiate Professorship, Jamie Buhr Fund, and Richard Nerod Fund. 


\section{Footnote}

Conflicts of Interest: The authors have no conflicts of interest to declare.

Ethical Statement: This study was approved by the Institutional Review Board at the University of Michigan, Michigan Medicine and was in compliance with Health Insurance Portability and Accountability Act regulations.

\section{References}

1. Estrera AL, Sandhu HK, Leake SS, et al. Early and late outcomes of acute type A aortic dissection with intramural hematoma. J Thorac Cardiovasc Surg 2015;149:137-42.

2. Sandhu HK, Tanaka A, Charlton-Ouw KM, et al. Outcomes and management of type A intramural hematoma. Ann Cardiothorac Surg 2016;5:317-27.

3. Uchida K, Imoto K, Karube N, et al. Intramural haematoma should be referred to as thrombosed-type aortic dissection. Eur J Cardiothorac Surg 2013;44:366-9; discussion 369.

4. Kitai T, Kaji S, Yamamuro A, et al. Clinical outcomes of medical therapy and timely operation in initially diagnosed type a aortic intramural hematoma: a 20 -year experience. Circulation 2009;120:S292-8.

5. Ho HH, Cheung CW, Jim MH, et al. Type A aortic intramural hematoma: clinical features and outcomes in Chinese patients. Clin Cardiol 2011;34:E1-5.

6. Kaji S, Akasaka T, Horibata Y, et al. Long-term prognosis of patients with type a aortic intramural hematoma. Circulation 2002;106:I248-52.

7. Evangelista A, Mukherjee D, Mehta RH, et al. Acute intramural hematoma of the aorta: a mystery in evolution. Circulation 2005;111:1063-70.

8. Harris KM, Braverman AC, Eagle KA, et al. Acute aortic intramural hematoma: an analysis from the International Registry of Acute Aortic Dissection. Circulation 2012;126:S91-6.

9. Hiratzka LF, Bakris GL, Beckman JA, et al. 2010 ACCF/ AHA/AATS/ACR/ASA/SCA/SCAI/SIR/STS/SVM guidelines for the diagnosis and management of patients with thoracic aortic disease. Circulation 2010;121:e266-369.

10. Sadamatsu K, Takase S, Sagara S, et al. Initial medical management in acute type A aortic dissection patients with a thrombosed false lumen in the ascending aorta combining intramural hematoma and retrograde dissection from the descending to the ascending aorta. Eur Heart J
Acute Cardiovasc Care 2018. [Epub ahead of print].

11. Watanabe S, Hanyu M, Arai Y, et al. Initial medical treatment for acute type a intramural hematoma and aortic dissection. Ann Thorac Surg 2013;96:2142-6.

12. Deeb GM, Williams DM, Bolling SF, et al. Surgical delay for acute type A dissection with malperfusion. Ann Thorac Surg 1997;64:1669-75; discussion 1675-7.

13. Patel HJ, Williams DM, Dasika NL, et al. Operative delay for peripheral malperfusion syndrome in acute type A aortic dissection: a long-term analysis. J Thorac Cardiovasc Surg 2008;135:1288-95; discussion 1295-6.

14. Yang B, Rosati CM, Norton EL, et al. Endovascular Fenestration/Stenting First Followed by Delayed Open Aortic Repair for Acute Type A Aortic Dissection With Malperfusion Syndrome. Circulation 2018;138:2091-103.

15. Yang B, Norton EL, Rosati CM, et al. Managing patients with acute type A aortic dissection and mesenteric malperfusion syndrome: 20-year experience. J Thorac Cardiovasc Surg 2019;158:675-87.e4.

16. Yu Y, Fei A, Wu Z, Wang H, et al. Aortic intramural hemorrhage: A distinct disease entity with mystery. Intractable Rare Dis Res 2017;6:87-94.

17. Centers for Disease Control and Prevention; National Center for Health Statistics. National Death Index. Accessed December 27, 2017. Available online: http:// www.cdc.gov/nchs/ndi/index.htm

18. Williams DM, Lee DY, Hamilton BH, et al. The dissected aorta: percutaneous treatment of ischemic complications-principles and results. J Vasc Interv Radiol 1997;8:605-25 .

19. Yang B, Norton EL, Hobbs R, et al. Short- and Longterm Outcomes of Aortic Root Repair and Replacement in Patients Undergoing Acute Type A Aortic Dissection Repair: 20-Year Experience. J Thorac Cardiovasc Surg 2019;157:2125-36.

20. Yang B, Malik A, Waidley V, et al. Short-term outcomes of a simple and effective approach to aortic root and arch repair in acute type A aortic dissection. J Thorac Cardiovasc Surg 2018;155:1360-70.e1.

21. Tanaka K, Morioka K, Li W, et al. Adventitial inversion technique without the aid of biologic glue or Teflon buttress for acute type A aortic dissection. Eur J Cardiothorac Surg 2005;28:864-9.

22. Rylski B, Bavaria JE, Milewski RK, et al. Long-term results of neomedia sinus valsalva repair in 489 patients with type A aortic dissection. Ann Thorac Surg 2014;98:582-8; discussion 588-9.

23. Yang B, Norton EL, Shih T, et al. Late Outcomes of Strategic Arch Resection in Acute Type A Aortic Dissection. 
J Thorac Cardiovasc Surg 2019;157:1313-21.e2.

24. Mohr-Kahaly S, Erbel R, Kearney P, et al. Aortic intramural hemorrhage visualized by transesophageal echocardiography: findings and prognostic implications. J Am Coll Cardiol 1994;23:658-64.

25. Tsai TT, Nienaber CA, Eagle KA. Acute aortic syndromes. Circulation 2005;112:3802-13.

26. Goldberg JB, Kim JB, Sundt TM. Current understandings and approach to the management of aortic intramural hematomas. Semin Thorac Cardiovasc Surg 2014;26:123-31.

27. Kitai T, Kaji S, Yamamuro A, et al. Detection of intimal defect by 64-row multidetector computed tomography in patients with acute aortic intramural hematoma. Circulation 2011;124:S174-8.

28. Estrera AL, Miller CC 3rd, Guajardo-Salinas G, et al. Update on blunt thoracic aortic injury: fifteen-year single-institution experience. J Thorac Cardiovasc Surg 2013;145:S154-8.

29. Tittle SL, Lynch RJ, Cole PE, et al. Midterm follow-up of penetrating ulcer and intramural hematoma of the aorta. J Thorac Cardiovasc Surg 2002;123:1051-9.

30. Coady MA, Rizzo JA, Elefteriades JA. Pathologic variants of thoracic aortic dissections. Penetrating atherosclerotic ulcers and intramural hematomas. Cardiol Clin 1999;17:637-57.

31. Chou AS, Ziganshin BA, Charilaou P, et al. Long-term behavior of aortic intramural hematomas and penetrating ulcers. J Thorac Cardiovasc Surg 2016;151:361-72, 373.e1.

32. Hata M, Hata H, Sezai A, et al. Optimal treatment strategy for type A acute aortic dissection with intramural hematoma. J Thorac Cardiovasc Surg 2014;147:307-11.

33. Geirsson A, Szeto WY, Pochettino A, et al. Significance of malperfusion syndromes prior to contemporary surgical repair for acute type A dissection: outcomes and need for additional revascularizations. Eur J Cardiothorac Surg 2007;32:255-62.

34. Girdauskas E, Kuntze T, Borger MA, et al. Surgical risk of preoperative malperfusion in acute type A aortic dissection. J Thorac Cardiovasc Surg 2009;138:1363-9.

35. Matsushita A, Fukui T, Tabata M, et al. Preoperative characteristics and surgical outcomes of acute intramural hematoma involving the ascending aorta: A propensity score-matched analysis. J Thorac Cardiovasc Surg 2016;151:351-8.
Cite this article as: Norton EL, Williams DM, Kim KM, Wu X, Khaja MS, Patel HJ, Deeb GM, Yang B. Management of malperfusion syndrome in acute type A aortic intramural hematoma. Ann Cardiothorac Surg 2019;8(5):540-550. doi: 10.21037/acs.2019.07.03 
Table S1 Comorbidities and clinical condition on admission of patients with or without malperfusion syndrome (only patients who underwent aortic repair)

\begin{tabular}{|c|c|c|c|c|}
\hline Admission variables & All patients $(n=79)$ & $\operatorname{MPS}(n=9)$ & No MPS $(n=70)$ & $P$ value \\
\hline Age at operation [years] & $64[56,73]$ & $63[58,73]$ & $64[56,73]$ & 0.95 \\
\hline Gender, male & $44[56]$ & $5[56]$ & 39 [56] & 1.0 \\
\hline CAD & 15 [19] & $1[11]$ & $14[20]$ & 0.67 \\
\hline History of MI & $5[6]$ & $0[0]$ & $5[7.1]$ & 1.0 \\
\hline Hypertension & 64 [81] & $8[89]$ & $56[80]$ & 1.0 \\
\hline COPD & $10[13]$ & 2 [22] & $8[11]$ & 0.32 \\
\hline Smoking status & & & & 0.48 \\
\hline Never smoker & 33 [42] & $2[22]$ & $31[44]$ & \\
\hline Former smoker & 27 [34] & $4[44]$ & 23 [33] & \\
\hline Current smoker & 19 [24] & $3[33]$ & $16[23]$ & \\
\hline Diabetes & $9[11]$ & $2[22]$ & $7[10]$ & 0.27 \\
\hline Chronic kidney disease & $0[0]$ & $0[0]$ & $0[0]$ & - \\
\hline History of CVA & $4[5]$ & $0[0]$ & $4[6]$ & 1.0 \\
\hline PVOD & 10 [13] & 2 [22] & $8[11]$ & 0.32 \\
\hline Connective tissue disorder & $3[4]$ & $0[0]$ & $3[4]$ & 1.0 \\
\hline \multicolumn{5}{|l|}{ Clinical condition on admission } \\
\hline Cardiogenic shock & $8[10]$ & $1[11]$ & $7[10]$ & 1.0 \\
\hline Ejection fraction & $56.8[55,60]$ & $55[55,60]$ & $60[55,60]$ & 0.38 \\
\hline Aortic insufficiency & & & & 0.91 \\
\hline None & $31[42]$ & $5[56]$ & $26[40]$ & \\
\hline Trace/trivial & $12[16]$ & $1[11]$ & $11[17]$ & \\
\hline Mild & $25[34]$ & $3[33]$ & 22 [34] & \\
\hline Moderate & $4[5]$ & $0[0]$ & $4[6]$ & \\
\hline Severe & 2 [3] & $0[0]$ & $2[3]$ & \\
\hline Acute MI & $1[1]$ & $1[11]$ & $0[0]$ & 0.11 \\
\hline Acute stroke & $2[3]$ & $1[11]$ & $1[1.4]^{*}$ & 0.22 \\
\hline Acute paralysis & $1[1]$ & $1[11]$ & $0[0]$ & 0.11 \\
\hline Acute renal failure & $4[5]$ & $3[33]$ & $1[1]$ & $<0.01$ \\
\hline Creatinine on admission (mg/dL) & $0.9[0.8,1.1]$ & $1.0[0.8,1.1]$ & $0.9[0.8,1.1]$ & 0.31 \\
\hline Malperfusion syndrome & $9[11]$ & 9 [100] & $0[0]$ & $<0.01$ \\
\hline Coronary & $1[1]$ & $1[11]$ & $0[0]$ & 0.11 \\
\hline Cerebral & $1[1]$ & $1[11]$ & $0[0]$ & 0.11 \\
\hline Spinal cord & $1[1]$ & $1[11]$ & $0[0]$ & 0.11 \\
\hline Celiac/hepatic & $1[1]$ & $1[11]$ & $0[0]$ & 0.11 \\
\hline Mesenteric & 2 [3] & 2 [22] & $0[0]$ & 0.01 \\
\hline Renal & $3[4]$ & $3[33]$ & $0[0]$ & $<0.01$ \\
\hline Lower extremity & $2[3]$ & 2 [22] & $0[0]$ & 0.01 \\
\hline IR & $7[9]$ & 7 [78] & $0[0]$ & $<0.01$ \\
\hline
\end{tabular}

Data presented as median (interquartile range) for continuous variables and number (percentage) for categorical variables. *, no MPS acute stroke: patient suffered anoxic brain injury due to cardiac arrest with ROSC preoperatively. MPS, malperfusion syndrome; CAD, coronary artery disease; MI, myocardial infarction; COPD, chronic obstructive pulmonary disease; CVA, cerebrovascular accident; PVOD, peripheral vascular occlusive disease; IR, interventional radiology. 


\begin{tabular}{|c|c|c|c|c|}
\hline Aortic repair & All patients ( $\mathrm{n}=79$ ) & MPS $(n=9)$ & No MPS $(n=70)$ & $P$ value \\
\hline TEVAR & $2[3]$ & $1[11]$ & $1[1]$ & 0.22 \\
\hline Open repair & 77 [97] & $8[89]$ & 69 [99] & 0.22 \\
\hline Aortic root procedure & & & & 0.76 \\
\hline None & 11 [14] & 2 [22] & $9[13]$ & \\
\hline Root replacement & $13[16]$ & 1 [11] & $12[17]$ & \\
\hline Root repair & $52[66]$ & $5[56]$ & $47[67]$ & \\
\hline AVR only & $1[1]$ & $0[0]$ & $1[1]$ & \\
\hline Ascending & 77 [97] & $8[89]$ & 69 [99] & 0.22 \\
\hline Aortic arch procedure & & & & 0.40 \\
\hline None & $3[4]$ & $0[0]$ & $3[4]$ & \\
\hline Hemiarch & $58[73]$ & $5[56]$ & $53[76]$ & \\
\hline Zone 1 arch & $3[4]$ & $0[0]$ & $3[4]$ & \\
\hline Zone 2 arch & $9[11]$ & $2[22]$ & $7[10]$ & \\
\hline Zone 3 arch & 4 [5] & $1[11]$ & $3[4]$ & \\
\hline Frozen elephant trunk & $4[5]$ & $0[0]$ & $4[6]$ & 1.0 \\
\hline \multicolumn{5}{|l|}{ Concomitant procedures } \\
\hline CABG & 7 [9] & $0[0]$ & $7[10]$ & 1.0 \\
\hline MV procedure & $0[0]$ & $0[0]$ & $0[0]$ & - \\
\hline TV procedure & $1[1]$ & $1[11]$ & $0[0]$ & 0.11 \\
\hline CPB time [min] & $210[170,246]$ & $226[179,250]$ & $210[169,238]$ & 0.57 \\
\hline Cross clamp time [min] & $125[108,164]$ & $133[126,202]$ & $121[105,160]$ & 0.15 \\
\hline HCA performed & 74 [94] & $8[89]$ & 66 [94] & 0.46 \\
\hline HCA time [min] & $32[27,40]$ & $32[27,53]$ & $32[27,39]$ & 0.52 \\
\hline Type of cerebral perfusion & & & & 0.21 \\
\hline ACP only & 39 [49] & $2[22]$ & 37 [53] & \\
\hline RCP only & $20[25]$ & $3[33]$ & $17[24]$ & \\
\hline Both ACP and RCP & $14[18]$ & $3[33]$ & $11[16]$ & \\
\hline Neither ACP nor RCP & 4 [5] & $0[0]$ & $4[6]$ & \\
\hline Intraoperative PRBC units & $2.5[0,5]$ & $2[0,3]$ & $3[0,6]$ & 0.72 \\
\hline 0 & 26 [33] & $3[33]$ & $23[33]$ & \\
\hline $1-2$ & $13[16]$ & $2[22]$ & $11[16]$ & \\
\hline $3-4$ & $15[19]$ & 2 [22] & $13[19]$ & \\
\hline$\geq 5$ & 24 [30] & 2 [22] & 22 [31] & \\
\hline
\end{tabular}

Data presented as median (interquartile range) for continuous variables and number (percentage) for categorical variables. MPS, malperfusion syndrome; TEVAR, thoracic endovascular aortic repair; AVR, aortic valve replacement; CABG, coronary artery bypass graft; MV, mitral valve procedure; TV, tricuspid valve procedure; CPB, cardiopulmonary bypass; HCA, hypothermic circulatory arrest; ACP, antegrade cerebral perfusion; RCP, retrograde cerebral perfusion; PRBC, packed red blood cell. 